\title{
On the Cohomological Derivation of Yang-Mills Theory in the Antifield Formalism
}

\author{
Ashkbiz Danehkar1,2 \\ ${ }^{1}$ Faculty of Physics, University of Craiova, Craiova, Romania \\ ${ }^{2}$ Present Address: Center for Astrophysics, Cambridge, MA, USA \\ Email: ashkbiz.danehkar@cfa.harvard.edu
}

How to cite this paper: Danehkar, A. (2017) On the Cohomological Derivation of Yang-Mills Theory in the Antifield Formalism. Journal of High Energy Physics, Gravitation and Cosmology, 3, 368-387. https://doi.org/10.4236/jhepgc.2017.32031

Received: January 17, 2017

Accepted: April 27, 2017

Published: April 30, 2017

Copyright (C) 2017 by author and Scientific Research Publishing Inc. This work is licensed under the Creative Commons Attribution International License (CC BY 4.0).

http://creativecommons.org/licenses/by/4.0/

\begin{abstract}
We present a brief review of the cohomological solutions of self-coupling interactions of the fields in the free Yang-Mills theory. All consistent interactions among the fields have been obtained using the antifield formalism through several order BRST deformations of the master equation. It is found that the coupling deformations halt exclusively at the second order, whereas higher order deformations are obstructed due to non-local interactions. The results demonstrate the BRST cohomological derivation of the interacting Yang-Mills theory.
\end{abstract}

\section{Keywords}

Yang-Mills Theory, BRST Symmetry, BRST Cohomology, Antifield Formalism

\section{Introduction}

Dirac's pioneering approach [1] [2] [3] has been used for constrained systems in quantum field theory [4] [5] [6]. This approach allowed us to construct the action in either Lagrangian or Hamiltonian forms [7] [8], while both of them are equivalent [9]. In this way, the Hamiltonian quantization is derived using canonical variables (coordinate and momentum) involving constrained dynamics [10]-[15]. Physical variables of a constrained system possess gauge invariance and locally independent symmetry. The gauge symmetry introduces some arbitrary time independent functions to the Hamilton's equations of motion. We notice that all canonical variables are not independent. Therefore, some conditions for canonical variables are required to be imposed, i.e., the first- and second-class constraints. Furthermore, the framework should be generalized to include both commutative (bosonic) and anticommutative (fermionic) variables in constrained systems. 
To generalize constrained systems for canonical conditions and (anti-)commutative variables, Becchi, Rouet, Stora [16] [17] [18], and Tyutin [19] developed the BRST formalism to extend the gauge symmetry in terms of the BRST differential and co-/homological classes. The aim was to replace the original gauge symmetry with the BRST symmetry. Noting that the gauge symmetry can be constructed from a nilpotent derivation, so the gauge action is invariant under a nilpotent symmetry, called the BRST symmetry. By replacing the original gauge symmetry with the BRST symmetry, antifield, ghosts, and antighosts are introduced for each gauge variable [20] [21]. It yields a generalized framework for solutions of the equations of motion [22] [23]. Moreover, BRST cohomology extended by the antifield formalism [23]-[30] allowed us to construct all consistent interactions among the fields using coupling deformations of the master equation [31] [32]. The BRST-antifield formalism appears as efficient mathematical tool to analyze the consistent interactions, and has been applied to many gauge models, e.g., Yang-Mills model [33], topological Yang-Mills model [34], 5-D topological BF model [35], and 5-D dual linearized gravity coupled to topological BF model [36].

In this paper, we briefly review the construction of all consistent interactions of the free Yang-Mills theory determined from all coupling deformations of the master equation. We see that the resulting action presents deformed structures of the gauge transformation and yields a commutator for it. In Section 2, the BRST differential and the antifield formalism are introduced. Section 3 introduces the consistent interactions among the fields. We consider the BRST coupling deformations of the master equations in the antifield formalism in Section 4. In Section 5, we demonstrate its application to the massless Yang-Mills theory by calculating all several order deformation of the master equation. Section 6 presents a conclusion.

\section{BRST Differential}

The gauge invariant in a phase space implies that the smooth phase space $C^{\infty}(P)$ is substituted by the smooth manifold of the constraint surface $C^{\infty}(\Sigma)$ while the elements of $C^{\infty}(\Sigma)$ vanish due to the longitudinal exterior derivative on manifold $\Sigma$. The manifold $\Sigma$, which is embedded in a phase space and a set of vectors tangent to $\Sigma$, and is closed on it, presents the definition of the gauge orbits. It manifests the presentation of a nilpotent derivation $s$, the so-called BRST differential, that includes an algebra involving $C^{\infty}(P)$, where the cohomology of $s$ indicates that the gauge transformations of the constraint surface $C^{\infty}(\Sigma)$ are constant along the gauge orbits (denoted by $\mathcal{G}$ ).

The reduced space, by taking $\Sigma$ over gauge orbits, denote by algebra $C^{\infty}(\Sigma / \mathcal{G})$, includes all variables of the gauge invariant. However, it is not possible to construct $C^{\infty}(\Sigma / \mathcal{G})$ from physical observables, as one cannot solve equations defining $\Sigma$ and trace the gauge orbits $\mathcal{G}$. Hence, the BRST symmetry should be used to reformulate the physical observables in a convenient approach. To construct the BRST differential $s$, two auxiliary derivations $\delta$ and 
$\gamma$ are introduced. The differential of the first derivation $\delta$ is called the Koszul-Tate differential that yields a resolution of the smooth manifold of the constraint surface $C^{\infty}(\Sigma)$. The second differential is called the longitudinal differential $\gamma$ along the gauge orbits in such its zeroth cohomology group provides the functions on the surface $\Sigma$ being constant along the gauge orbits $\mathcal{G}$. Hence, the BRST differential $s$ is decomposed into [22] [23] [27]

$$
s=\delta+\gamma,
$$

whose cohomology is equal to the cohomology of the longitudinal differential $\gamma$, while the Koszul-Tate differential $\delta$ restricts it to the constrains surface $C^{\infty}(\Sigma)$. Note that the BRST symmetry acts as a general odd derivation on the original fields and some auxiliary fields (antifields and ghosts), which are equipped for any $X$ and $Y$ with Grassmann parity $\varepsilon_{X}$ and $\varepsilon_{Y}$ :

$$
\begin{gathered}
s(X Y)=X(s Y)+(-1)^{\varepsilon_{Y}}(s X) Y, \quad \text { (Leibniz rule) } \\
s^{2}=0 . \quad \text { (nilpotency) }
\end{gathered}
$$

where $\varepsilon_{X}=0$ or 1 for bosonic (commutative) or fermionic (anticommutative) variable $X$, respectively.

Any nilpotent derivation has a degree in a $N$-grading space denoted by

$$
\operatorname{deg}(s)= \pm 1 \text {. }
$$

The positive degree of the differential $s$ increases the grading while the negative degree decreases it, i.e. $s\left(X_{n}\right) \subset X_{n \pm 1}$ depending on the degree of the differential operator. The grading of $s$ is the so-called ghost number $(\mathfrak{g h})$, equal to one, consists of the pureghost number $(\mathfrak{p g h})$ and the antighost number $\left({ }^{\mathfrak{a} \mathfrak{g h}}\right)$ :

$$
\mathfrak{g h}(X)=\mathfrak{p} \mathfrak{g h}(X)-\mathfrak{a g h}(X),
$$

with the following property

$$
\mathfrak{g h}(X Y)=\mathfrak{g h}(X)+\mathfrak{g h}(Y),
$$

where the operators $\mathfrak{p g h}$ and $\mathfrak{a g h}$ stand for the pureghost and antighost numbers, respectively. For the Koszul-Tate differential $\delta$ and the longitudinal differential $\gamma$, we get:

$$
\mathfrak{p} \mathfrak{g} \mathfrak{h}(\delta)=0, \quad \mathfrak{a} \mathfrak{g} \mathfrak{h}(\delta)=-1, \quad \mathfrak{p} \mathfrak{g} \mathfrak{h}(\gamma)=1, \quad \mathfrak{a} \mathfrak{g} \mathfrak{h}(\gamma)=0,
$$

such $\mathfrak{g} \mathfrak{h}(s)=\mathfrak{g h}(\delta)=\mathfrak{g h}(\gamma)=1$. The differentials $\delta$ and $\gamma$ increase the ghost number by one unit. The differential $\delta$ reduces the antighost number, but maintains the pureghost number, whereas the differential $\gamma$ increases the pureghost number, but maintains the antighost number.

The cohomology algebra of the differential $s$ is $H(s)=\operatorname{Ker} s / \operatorname{Im} s$, where the elements of the kernel subspace, $\operatorname{Ker} s$, are closed and vanish via the differential $s$ :

$$
s a=0, \quad a \in \operatorname{Ker} s,
$$

while the elements of its image subspace, $\operatorname{Im} s$, are exact: 


$$
s b=a, \quad a \in \operatorname{Im} s .
$$

The cohomology algebra of $s$, denoted by $H^{k}(s)$ ( $k$ is a cohomology degree), exists if its degree is positive, whereas its homology algebra, denoted by $H_{k}(s)$, has a negative degree. The co-/homology with the grading algebra then reads as follows

$$
\begin{aligned}
& \operatorname{deg}(s)=+1 \rightarrow H^{k}(s)=\underset{n \in \mathbb{N}}{\oplus} H^{n}(s), \\
& \operatorname{deg}(s)=-1 \rightarrow H_{k}(s)=\bigoplus_{n \in \mathbb{N}} H_{n}(s) .
\end{aligned}
$$

If the co-/homology $H^{k}(s)$ is zero, the differential $s$ is called to be acyclic in a degree of $k$.

The zeroth cohomology group of the BRST differential $H^{0}(s)$ leads to Equation (3), the essential aspect of the BRST symmetry, that implies the vanishing squares of its derivations $\delta$ and $\gamma$ :

$$
\delta^{2}=0, \quad \gamma^{2}=0
$$

and also their anticommutation:

$$
\gamma \delta+\delta \gamma=0
$$

It means that the Koszul-Tate differential $\delta$ commutes with the longitudinal differential $\gamma$.

The generator of the Koszul-Tate complex may be chosen in an equal number of freedom as the generator of the longitudinal exterior complex. It follows that they are canonically conjugate in the extended space of original and new generators of $\delta$ and $\gamma$. This implies that the BRST transformation maintains a canonical transformation in the BRST complex space $\mathbb{C}\left[x^{k}\right]$ through a bracket structure:

$$
s X=[X, \Omega], \quad \forall X \in \mathbb{C}\left[x^{k}\right],
$$

which is called the Poisson bracket and defined as follows:

$$
[X, Y] \equiv \frac{\partial X}{\partial q^{k}} \frac{\partial Y}{\partial p_{k}}-\frac{\partial X}{\partial p_{k}} \frac{\partial Y}{\partial q^{k}}
$$

where $q_{k}$ and $p_{k}$ are positions and canonical momenta of a Hamiltonian system, respectively.

Equation (13) represents the BRST symmetry in the Hamiltonian formalism. The choice of $s$ as canonical transformation manifests the BRST symmetry where the canonical variables remain unchanged under transformation. The fermionic charge $\Omega$ is called the BRST generator for the Hamiltonian formalism. Applying the Jacobi identity to the Poisson bracket and the nilpotency definition of the BRST differential yields:

$$
[\Omega, \Omega]=0 \text {, }
$$

which is the master equation of the BRST generator in the Hamiltonian formalism.

\section{Consistent Interactions}

To understand the consistent interactions among fields with a gauge freedom, we begin our study with a Lagrangian action: 


$$
S_{0}^{L}\left[\phi^{\alpha_{0}}\right]=\int \mathrm{d}^{D} x \mathcal{L}_{0}\left(\phi^{\alpha_{0}}, \partial_{\mu} \phi^{\alpha_{0}}, \partial_{\mu} \partial_{\nu} \phi^{\alpha_{0}}, \cdots, \partial_{\mu_{1}} \partial_{\mu_{2}} \cdots \partial_{\mu_{k}} \phi^{\alpha_{0}}\right),
$$

where the action $S_{0}^{L}\left[\phi^{\alpha_{0}}\right]$ is local functional of the fields $\phi^{\alpha_{0}}$ and their Lorentz covariant derivatives.

The equations of motion then read $\delta S_{0}^{L} / \delta \phi^{\alpha_{0}}(x)=0$, where $\delta S_{0}^{L} / \delta \phi^{\alpha_{0}}$ is functional derivatives. The action $S_{0}^{L}\left[\phi^{\alpha_{0}}\right]$ possesses generic free gauge symmetries

$$
\delta_{\varepsilon} \phi^{\alpha_{0}}=Z_{\alpha_{1}}^{\alpha_{0}} \varepsilon^{\alpha_{1}},
$$

The equations of motion is then determined from the action principle: $\delta_{\varepsilon} S_{0}^{L}\left[\phi^{\alpha_{0}}\right]=0$.

Let consider the deformations of the action in such a way

$$
S_{0}^{L}\left[\phi^{\alpha_{0}}\right] \rightarrow S^{L}\left[\phi^{\alpha_{0}}\right]=S_{0}^{L}\left[\phi^{\alpha_{0}}\right]+\lambda S_{1}^{L}\left[\phi^{\alpha_{0}}\right]+\lambda^{2} S_{2}^{L}\left[\phi^{\alpha_{0}}\right]+\cdots,
$$

that implies the deformation of gauge symmetries as

$$
Z_{\alpha 1}^{\alpha_{0}} \rightarrow \bar{Z}_{\alpha 1}^{\alpha_{0}}=Z_{\alpha 1}^{\alpha_{0}}+\lambda Z_{\alpha 1}^{\alpha_{0}}+\lambda^{2} Z_{\alpha 1}^{(2)}+\cdots
$$

This provides the deformed gauge transformations:

$$
\frac{\delta S^{L}}{\delta \phi^{\alpha_{0}}} \bar{Z}_{\alpha_{1}}^{\alpha_{0}}=0
$$

Equation (18) and Equation (19) lead to the following expression:

$$
\left(\frac{\delta S_{0}^{L}}{\delta \phi^{\alpha_{0}}}+\lambda \frac{\delta S_{1}^{L}}{\delta \phi^{\alpha_{0}}}+\lambda^{2} \frac{\delta S_{2}^{L}}{\delta \phi^{\alpha_{0}}}+\cdots\right)\left(Z_{\alpha_{1}}^{\alpha_{0}}+\lambda Z_{\alpha 1}^{\alpha_{0}}+\lambda^{2} Z_{\alpha 1}^{\alpha_{0}}+\cdots\right)=0 .
$$

Hence, the deformations by their orders are as follows:

$$
\begin{cases}\lambda^{0}: & \frac{\delta S_{0}^{L}}{\delta \phi^{\alpha_{0}}} Z_{\alpha_{1}}^{\alpha_{0}}=0, \\ \lambda^{1}: & \frac{\delta S_{0}^{L}}{\delta \phi^{\alpha_{0}}} Z_{\alpha 1}^{\alpha_{0}}+\frac{\delta S_{1}^{L}}{\delta \phi^{\alpha_{0}}} Z_{\alpha_{1}}^{\alpha_{0}}=0, \\ \lambda^{2}: & \frac{\delta S_{0}^{L}}{\delta \phi^{\alpha_{0}}} Z_{\alpha 1}^{\alpha_{0}}+\frac{\delta S_{1}^{L}}{\delta \phi^{\alpha_{0}}} Z_{\alpha 1}^{\alpha_{0}}+\frac{\delta S_{2}^{L}}{\delta \phi^{\alpha_{0}}} Z_{\alpha_{1}}^{\alpha_{0}}=0, \\ \vdots & \vdots\end{cases}
$$

which define the deformed gauge transformations that close on-shell for the interacting action, the so-called consistent interactions, while the original gauge transformations are reducible [28].

Assume that the gauge fields of consistent interactions are trivially defined to be the following sum:

$$
\phi^{\alpha_{0}} \rightarrow \bar{\phi}^{\alpha_{0}}=\phi^{\alpha_{0}}+\lambda F^{\alpha_{0}}\left[\phi^{\beta_{0}}\right]+\lambda^{2} F^{\alpha_{0}}\left[\phi^{\beta_{0}}\right]+\cdots,
$$

we then obtain

$$
\begin{aligned}
S_{0}^{L}\left[\phi^{\alpha_{0}}\right] \rightarrow S^{L}\left[\phi^{\alpha_{0}}\right] & =S_{0}^{L}\left[\phi^{\alpha_{0}}+\lambda F^{\alpha_{0}}+\cdots\right] \\
= & S_{0}^{L}\left[\phi^{\alpha_{0}}\right]+\lambda \frac{\delta S_{0}^{L}}{\delta \phi^{\alpha_{0}}} F_{1}^{\alpha_{0}} \\
& +\lambda^{2}\left(\frac{\delta^{2} S_{0}^{L}}{\delta \phi^{\alpha_{0}} \delta \phi^{\beta_{0}}} F_{1}^{\alpha_{0}} F_{1}^{\beta_{0}}+\frac{\delta^{2} S_{0}^{L}}{\delta\left(\phi^{\alpha_{0}}\right)^{2}} F_{2}^{\alpha_{0}}\right)+\cdots
\end{aligned}
$$


which does not manifest an exact interacting theory. A theory is strict if the consistent deformations are merely proportional to its free theory action $S_{0}^{L}\left[\phi^{\alpha_{0}}\right]$ up to the redefinition of the gauge fields. Thus, the interaction is formulated as follows:

$$
S_{0}^{L}\left[\phi^{\alpha_{0}}\right] \rightarrow S^{L}\left[\phi^{\alpha_{0}}\right]=\left(1+\mathcal{Q}_{1} \lambda+\left(\mathcal{Q}_{2} \lambda\right)^{2}+\cdots\right) S_{0}^{L}\left[\phi^{\alpha_{0}}\right]
$$

where charges $\mathcal{Q}_{k}$ in the $k$ order of the coupling constants $\lambda^{k}$ are given by

$$
\begin{gathered}
\mathcal{Q}_{1} \equiv \frac{\delta}{\delta \phi^{\alpha_{0}}} F_{1}^{\alpha_{0}}, \\
\mathcal{Q}_{2}^{2} \equiv \frac{\delta^{2}}{\delta \phi^{\alpha_{0}} \delta \phi^{\beta_{0}}} F_{1}^{\alpha_{0}} F_{1}^{\beta_{0}}+\frac{\delta^{2}}{\delta\left(\phi^{\alpha_{0}}\right)^{2}} F_{2}^{\alpha_{0}},
\end{gathered}
$$

It represents the unperturbed action by charges of the coupling constants.

\section{BRST Deformations of the Master Equation}

Let us consider the gauge transformation defined by the Equation (17). The classical fields $\phi^{\alpha_{0}}$ possesses the ghost number zero. It implies an ghost $\eta^{\alpha_{1}}$ associated to ghost number one, as well as the one-level ghost of ghost $\eta^{\alpha_{2}}$ have number two, etc., i.e.

$$
\eta^{A}=\left\{\eta^{\alpha_{1}}, \cdots, \eta^{\alpha_{k}}\right\}
$$

which have the following ghost numbers, $\mathfrak{g h}$, and Grassmann parities, $\varepsilon$ :

$$
\mathfrak{g h}\left(\eta^{\alpha_{k}}\right)=k, \quad \varepsilon\left(\eta^{\alpha_{k}}\right)=k(\bmod 2) .
$$

It also implies antifields $\phi_{\alpha_{0}}^{*}$ and antighosts $\eta_{A}^{*}$ of opposite Grassmann parity with the following ghost numbers, $\mathfrak{g h}$, and Grassmann parities, $\varepsilon$, respectively:

$$
\begin{gathered}
\mathfrak{g h}\left(\phi_{\alpha_{0}}^{*}\right)=-\mathfrak{g} \mathfrak{h}\left(\phi^{\alpha_{0}}\right)-1, \quad \varepsilon\left(\phi_{\alpha_{0}}^{*}\right)=\varepsilon\left(\phi^{\alpha_{0}}\right)+1(\bmod 2), \\
\mathfrak{g h}\left(\eta_{\alpha_{k}}^{*}\right)=-(k+1), \quad \varepsilon\left(\eta_{\alpha_{k}}^{*}\right)=k+1(\bmod 2) .
\end{gathered}
$$

The presentation of the gauge variables is therefore provided by

$$
\Phi^{A}=\left\{\phi^{\alpha_{0}}, \eta^{A}\right\}, \quad \Phi_{A}^{*}=\left\{\phi_{\alpha_{0}}^{*}, \eta_{A}^{*}\right\},
$$

where a set of fields $\Phi^{A}$ includes the original fields, the ghost, and the ghosts of ghosts, and $\Phi_{A}^{*}$ includes the their corresponding antifields.

The BRST symmetry is a canonical transformation, and defined by an antibracket structure:

$$
\bar{s} X \equiv(X, S),
$$

where $S$ is the canonical generators, and the antibracket (see appendix 7.1) is defined in the space of fields $\Phi^{A}$ and antifields $\Phi_{A}^{*}$ as follows [24]:

$$
(X, Y) \equiv \frac{\partial_{r} X}{\partial \Phi^{A}} \frac{\partial_{l} Y}{\partial \Phi_{A}^{*}}-\frac{\partial_{r} X}{\partial \Phi_{A}^{*}} \frac{\partial_{l} Y}{\partial \Phi^{A}} .
$$


The Grassmann parity and ghost number of the antibracket are, respectively:

$$
\begin{gathered}
\varepsilon(X, Y)=\varepsilon_{X}+\varepsilon_{Y}+1(\bmod 2), \\
\mathfrak{g h}(X, Y)=\mathfrak{g h}(X)+\mathfrak{g h}(Y)+1 .
\end{gathered}
$$

The antifields are now considered as mathematical tool to construct the BRST formalism. The solution can be interpreted as source coefficient for BRST transformation, i.e., an effective action in the theory.

The fields and antifields establish the solution $S\left[\Phi^{A}, \Phi_{A}^{*}\right]$ of the classical master equation for consistent interactions [31],

$$
S=S_{0}+\lambda S_{1}+\lambda^{2} S_{2}+\cdots
$$

Section 2 presented the master Equation (15) of the BRST generator in the Hamiltonian formalism. The gauge structure is now constructed through the solution $S$ of the master equation in the antifield formalism by [24] [25] [34]

$$
(S, S)=0 \text {. }
$$

This shows the consistency of the gauge transformations. The master Equation (36) includes the closure of the gauge transformations, the higher-order gauge identities, and the Noether identities. The master equation maintains the consistent specifications on $S_{0}$ and $Z_{\alpha_{1}}^{\alpha_{0}}$.

Substituting the definition (35) into the master Equation (36) yields

$$
\left(S_{0}+\lambda S_{1}+\lambda^{2} S_{2}+\cdots, S_{0}+\lambda S_{1}+\lambda^{2} S_{2}+\cdots\right)=0 .
$$

We then derive

$$
\left\{\begin{array}{cc}
\lambda^{0}: & \left(S_{0}, S_{0}\right)=0, \\
\lambda^{1}: & \left(S_{0}, \lambda S_{1}\right)+\left(\lambda S_{1}, S_{0}\right)=0, \\
\vdots & \vdots
\end{array}\right.
$$

which are simplified as follows [31] [36] [37] [38]

$$
\begin{gathered}
\left(S_{0}, S_{0}\right)=0, \\
2\left(S_{0}, S_{1}\right)=0, \\
2\left(S_{0}, S_{2}\right)+\left(S_{1}, S_{1}\right)=0, \\
\left(S_{0}, S_{3}\right)+\left(S_{1}, S_{2}\right)=0, \\
2\left(S_{0}, S_{4}\right)+2\left(S_{1}, S_{3}\right)+\left(S_{2}, S_{2}\right)=0, \\
\left(S_{0}, S_{5}\right)+\left(S_{1}, S_{4}\right)+\left(S_{2}, S_{3}\right)=0,
\end{gathered}
$$

the so-called deformations of the master equation [31] [32].

The Equation (40) implies that $S_{1}$ is a cocycle for the free differential defined by $s \equiv\left(\cdot, S_{0}\right)$, i.e., $S_{1}$ is a coboundary, $S_{1}=\left(B_{1}, S_{0}\right)$. The Equation (39) hence corresponds to $s^{2}=0$. The Equation (41) indicates that $\left(S_{1}, S_{1}\right)$ is trivial in $H^{1}(s)$, and $H^{0}(s)$ is mapped trivially into $H^{1}(s)$ by the antibracket. Furthermore, the higher orders $H^{0}(s)$ mapped into $H^{1}(s)$ are trivial, and provide the existence of the terms $S_{3}, S_{4}$, etc, up to an element of $H^{0}(s)$. So, the 
$k$ orders $\lambda^{k}$ freely link the interaction of an arbitrary element of $H^{0}(s)$.

The free gauge invariant action $S_{0}^{L}$ and the gauge transformations can be retrieved from

$$
S_{0}=S_{0}^{L}+\phi_{\alpha_{0}}^{*} Z_{\alpha_{1}}^{\alpha_{0}} \eta^{\alpha_{1}}+\cdots
$$

by setting

$$
S_{0}^{L}=S_{0}\left[\Phi^{A}, \Phi_{A}^{*}=0\right]
$$

It provides the solution $S_{0}$ of the classical master equation for field gauge symmetries,

$$
\left(S_{0}, S_{0}\right)=0
$$

The BRST differential $s$ is now defined by $S_{0}$ through the antibracket,

$$
s X \equiv\left(X, S_{0}\right)
$$

Using the definitions (48), the deformations of the master equation are rewritten as follows:

$$
\begin{array}{ll}
\lambda^{1}: & 2 s S_{1}=0 \\
\lambda^{2}: & \left(S_{1}, S_{1}\right)+2 s S_{2}=0 \\
\lambda^{3}: & \left(S_{1}, S_{2}\right)+s S_{3}=0 \\
\lambda^{4}: & 2\left(S_{1}, S_{3}\right)+\left(S_{2}, S_{2}\right)+2 s S_{4}=0 \\
\lambda^{5}: & \left(S_{1}, S_{4}\right)+\left(S_{2}, S_{3}\right)+s S_{5}=0 \\
\vdots & \vdots
\end{array}
$$

which are the deformations of the master equation in terms of the BRST differential $s$.

\section{BRST Cohomology of the Free Yang-Mills Theory}

Let us consider a set of $N$ potentials $A_{\mu}^{a}$ described by the abelian action in terms of the free (massless) Lagrangian action

$$
S_{0}^{L}\left[A_{\mu}^{a}\right]=\int \mathrm{d}^{D} x\left(-\frac{1}{4} F_{\mu \nu}^{a} F_{a}^{\mu \nu}\right), a=1, \cdots, N, N \in \mathbb{N},
$$

where $A_{\mu}^{a}$ is the abelian field potential, $D$ is the spacetime dimension, strictly $D>2$, since the theory has no local degree of freedom in two dimensions, and the abelian field strengths $F_{\mu \nu}^{a}$ is defined by

$$
F_{\mu \nu}^{a} \equiv \partial_{\mu} A_{v}^{a}-\partial_{\nu} A_{\mu}^{a}=\frac{\partial A_{v}^{a}}{\partial x^{\mu}}-\frac{\partial A_{\mu}^{a}}{\partial x^{v}}
$$

in such a way

$$
F_{a}^{\mu v}=\sigma^{\mu \alpha} \sigma^{\nu \beta} k_{a b} F_{\alpha \beta}^{b},
$$

where $\sigma^{\mu \alpha}=\operatorname{diag}(-1,1, \cdots, 1)$ is the $S O(1, D-1)$ invariant flat metric in Minkowski space with the particular hermitian representation of the Clifford al- 
gebra $\left\{\gamma^{\mu}, \gamma^{\nu}\right\}=2 \sigma^{\mu \nu}$, and $k_{a b}$ is a given symmetric invertible matrix with following properties

$$
k_{(a b)}=k_{a b}=k_{b a}, \quad k^{a b} k_{b c}=\delta^{a}{ }_{c}, \quad a, b, c=1, \cdots, N .
$$

The gauge transformation with the free equation of motion,

$$
\frac{\delta S_{0}^{L}}{\delta A_{\mu}^{a}}=\partial_{v} F_{a}^{v \mu}=0
$$

manifests an irreducible transformation by

$$
\delta_{\varepsilon} A_{\mu}^{a}=\partial_{\mu} \varepsilon^{a},
$$

while

$$
\delta_{\varepsilon} F_{\mu \nu}^{a}=\partial_{\mu} \partial_{\nu} \varepsilon^{a}-\partial_{\nu} \partial_{\mu} \varepsilon^{a}=0 .
$$

The differential operator $\partial_{\mu}$ is determined by the structure $Z_{\alpha_{1}}^{\alpha_{0}}$ of the gauge transformations of an abelian algebra. The action (50) is close according to an abelian algebra, and invariant under the gauge transformation (55). The gauge invariant (55) eliminates unphysical terms, i.e. the longitudinal and temporal degrees of freedom.

The implementation of the BRST transformation in the minimal sector provides the field $A_{\mu}^{a}$, its ghost $\eta^{a}$, and their antifields $A_{a}^{* \mu}$ and $\eta_{a}^{*}$ with the respective Grassmann parities, antighost, pureghost, and (total) ghost numbers,

\begin{tabular}{c|c|c|c|c}
$Z$ & $A_{\mu}^{a}$ & $A_{a}^{* \mu}$ & $\eta^{a}$ & $\eta_{a}^{*}$ \\
\hline$\varepsilon(Z)$ & 0 & 1 & 1 & 0 \\
$\mathfrak{a g h}(Z)$ & 0 & 1 & 0 & 2 \\
$\mathfrak{p g} \mathfrak{g}(Z)$ & 0 & 0 & 1 & 0 \\
$\mathfrak{g h}(Z)$ & 0 & -1 & 1 & -2
\end{tabular}

which can schematically be illustrated:

$$
\begin{array}{ccccc}
\varepsilon=0 & A_{\mu}^{a} & & \delta_{\varepsilon} & \partial_{\mu} \varepsilon^{a} \\
& & & & \\
& & \downarrow & & \\
& & \searrow & \\
\varepsilon=1 & A_{a}^{*} & & \\
& & & \eta^{a} \\
& & & g h=1 \\
& & & \downarrow \\
\varepsilon=0 & & & \eta_{a}^{*} \\
& & & & \\
& & & \\
& & & &
\end{array}
$$

We calculate the BRST-differential $s$ that decomposes into the sum of two differentials, the Koszul-Tate differential $\delta$ and the longitudinal differential $\gamma$ along the gauge orbits. Both $\delta$ and $\gamma$ are derivations, and commute with $\partial_{\mu}$, and acting on $A_{\mu}^{a}, A_{a}^{* \mu}, \eta^{a}$, and $\eta_{a}^{*}$ via [33] [39]

\begin{tabular}{c|c|c}
$Z$ & $\delta Z$ & $\gamma Z$ \\
\hline$A_{\mu}^{a}$ & 0 & $\partial_{\mu} \eta^{a}$ \\
$A_{a}^{* \mu}$ & $-\frac{\delta S_{0}^{L}}{\delta A_{\mu}^{a}}=-\partial_{\nu} F_{a}^{\nu \mu}$ & 0 \\
$\eta^{a}$ & 0 & 0 \\
$\eta_{a}^{*}$ & $-\partial_{\mu} A_{a}^{* \mu}$ & 0
\end{tabular}


The classical master Equation (47) of the action (50) holds the minimal solution (45) in such a way

$$
S_{0}=S_{0}^{L}\left[A_{\mu}^{a}\right]+\int \mathrm{d}^{D} x A_{a}^{* \mu} \partial_{\mu} \eta^{a} .
$$

\subsection{First-Order Deformation}

We now consider the deformed solution of the master equation for the action (50) smoothly in the coupling constant $\lambda$ that brings to the solution (58), while the coupling constant $\lambda$ vanishes. In Section 4 , we noticed that the first-order deformation $\left(\lambda^{1}\right)$ of the master equation satisfies the solution $s S_{1}=0$, where $S_{1}$ is bosonic (commutative) function with ghost number zero.

Let us assume

$$
S_{1}=\int \mathrm{d}^{D} x a
$$

where $a$ is a local function. Then, the first-order deformation, $s S_{1}=0$, takes the local form

$$
\begin{gathered}
\int \mathrm{d}^{D} x s a=0 \rightarrow s a=(\delta a+\gamma a)=\partial_{\mu} j^{\mu} \\
\mathfrak{g h}(a)=0, \quad \varepsilon(a)=0,
\end{gathered}
$$

where $j^{\mu}$ is a local current that manifests the non-integrated density of the first-order deformation corresponding to the local cohomology of $s$ in ghost number zero, $a \in H^{0}(s \mid d)$, where $d$ is the exterior spacetime differential.

To evaluate Equation (60), we assume

$$
\begin{gathered}
a=\sum_{i=0}^{I} a_{i}, \quad \mathfrak{a} \mathfrak{g} \mathfrak{h}\left(a_{i}\right)=i, \quad \mathfrak{g} \mathfrak{h}\left(a_{i}\right)=0, \quad \varepsilon\left(a_{i}\right)=0, \quad \forall i=0, \cdots, I, \\
j^{\mu}=\sum_{i=0}^{I} \stackrel{(i)}{j^{\mu}}, \quad \mathfrak{a} \mathfrak{g} \mathfrak{h}\left(\stackrel{(i)}{j^{\mu}}\right)=i, \quad \mathfrak{g} \mathfrak{h}\left(\stackrel{(i)}{j^{\mu}}\right)=0, \quad \varepsilon\left({ }^{\mu} j^{\mu}\right)=0,
\end{gathered}
$$

where $j^{\mu}$ are some local currents. Substituting (62) and (63) into (60) yields

$$
\sum_{i=0}^{I} \delta a_{i}+\sum_{i=0}^{I} \gamma a_{i}=\sum_{i=0}^{I} \partial_{\mu}{ }^{(i)} j^{\mu},
$$

obviously

$$
\mathfrak{a} \mathfrak{g h}\left(\delta a_{i}\right)=i-1, \quad \mathfrak{a} \mathfrak{g} \mathfrak{h}\left(\gamma a_{i}\right)=i .
$$

They can be decomposed on the several orders of the antighost number:

\begin{tabular}{c|c}
$\mathfrak{a g h}(Z)$ & $Z$ \\
\hline$I$ & $\gamma a_{I}=\partial_{\mu} \dot{j}^{\mu}$, \\
$I-1$ & $\delta a_{I}+\gamma a_{I-1}=\partial_{\mu}{ }_{(I-1)}^{\mu}$, \\
$k$ & $\delta a_{k+1}+\gamma a_{k}=\partial_{\mu} j^{\mu}, \quad k=0, \cdots, I-2$
\end{tabular}

The positive antighost number are strictly given as replacement for the first expression [35]: 


$$
\gamma a_{I}=0, \quad I>0 \rightarrow a_{I} \in H^{I}(\gamma) .
$$

To proof it, let us consider $e^{I}$ as the elements with pureghost number $I$ of a basis in the polynomial space. The generic solution of (67) then takes the form

$$
a_{I}=\alpha_{I} e^{I},
$$

while

$$
\mathfrak{a g h}\left(\alpha_{I}\right)=I, \quad \mathfrak{p g} \mathfrak{g h}\left(e^{I}\right)=I .
$$

The objects $\alpha_{I}$ obviously are nontrivial in $H^{0}(\gamma)$, the so-called invariant polynomials. In other words, the strict positive antighost numbers provide trivially the cohomology of the exterior differential $\gamma$ in the space of invariant polynomials $\alpha_{I}$. Hence, $\gamma a=\partial_{\mu} j^{\mu}$ reduces to $\gamma a=0$ (see [35] for general proof).

Moreover, $a_{I}$ may exclusively be reduced to $\gamma$-exact terms

$$
a_{I}=\gamma b_{I},
$$

corresponding to a trivial definition, which states $a_{I}=0$. This result is obviously given by the second-order nilpotency of $\gamma$ that implies the unique solution of (67) up to $\gamma$-exact contributions, i.e.

$$
\begin{array}{cl} 
& a_{I} \rightarrow a_{I}+\gamma b_{I}, \\
\mathfrak{a} \mathfrak{g h}\left(b_{I}\right)=I, & \mathfrak{p g h h}\left(b_{I}\right)=I-1, \quad \varepsilon\left(b_{I}\right)=1 .
\end{array}
$$

Hence, the non-triviality of the first-order deformation $a_{I}$ requires the cohomology of the exterior longitudinal derivative $\gamma$ in pureghost number equal to $I$, i.e. $a_{I} \in H^{I}(\gamma)$. To solve (66), it is necessary to provide the cohomology of $\gamma$ and $\delta, H(\gamma)$ and $H(\delta \mid d)$ :

$$
\delta a_{I}=\partial_{\mu} m_{I}^{\mu} \rightarrow a_{I} \in H_{I}(\delta \mid d),
$$

where

$$
H_{I}(\delta \mid d)=\left\{a \mid \mathfrak{a} \mathfrak{g h}(a)=I, \delta a=\partial_{\mu} m^{\mu}\right\} / N .
$$

For an irreducible linear situation, where gauge generators are field independent, we assume that

$$
H_{I}(\delta \mid d)=0, \quad I>2 .
$$

where $H_{I}(\delta \mid d)$ manifests the local cohomology of the Koszul-Tate differential $\delta$, while antighost number is $I$ and pureghost number vanishes. In this case $(I=2)$, we obtain

$$
\left\{\begin{array}{l}
\gamma a_{2}=0, \\
\delta a_{2}+\gamma a_{1}=\partial_{\mu}{ }^{\mu} j^{\mu}, \\
\delta a_{1}+\gamma a_{0}=\partial_{\mu} j^{\mu}
\end{array} .\right.
$$

The first-order deformation up to antighost number two are:

$$
a=a_{0}+a_{1}+a_{2} .
$$

The $a_{2}$ is generated by arbitrarily smooth functions in the form (68), with 
$\alpha_{2}$ from $H_{2}^{\text {inv }}(\delta \mid d)$ and $e^{2}$ denote the elements with pureghost number two of a basis in the polynomial space, i.e.,

$$
a_{2} \in H_{2}^{\text {inv }}(\delta \mid d) \rightarrow \mathfrak{a g h}\left(\alpha_{2}\right)=2, \quad \mathfrak{p g h}\left(e^{2}\right)=2,
$$

where $H_{I}^{\text {inv }}(\delta \mid d)$ is the local cohomology of the Koszul-Tate differential $\delta$ with antighost number $I$ in the invariant polynomial space.

We now consider the Koszul-Tate differential $\delta$ and the exterior longitudinal differential $\gamma$ in the action (58):

$$
\begin{gathered}
\delta A_{\mu}^{a}=\delta \eta^{a}=0, \quad \delta A_{a}^{* \mu}=-\partial_{\nu} F_{a}^{\nu \mu}, \quad \delta \eta_{a}^{*}=-\partial_{\mu} A_{a}^{* \mu}, \\
\gamma A_{\mu}^{a}=\partial_{\mu} \eta^{\alpha}, \quad \gamma A_{a}^{* \mu}=\gamma \eta^{a}=\gamma \eta_{a}^{*}=0 .
\end{gathered}
$$

The local cohomology of the exterior longitudinal derivative $\gamma$ in pureghost number one, $H^{1}(\gamma)$, has one ghost $\eta^{a}$, while $H^{2}(\gamma)$ has two ghosts $\eta^{a} \eta^{b}$, i.e.

$$
\left\{\eta^{a}\right\} \in H^{1}(\gamma), \quad\left\{\eta^{a} \eta^{b}\right\} \in H^{2}(\gamma)
$$

From (79), we then solve

$$
\gamma a_{2}=0,
$$

by

$$
a_{2}=\frac{1}{2} \eta_{a}^{*} f_{b c}^{a} \eta^{b} \eta^{c},
$$

where $f_{b c}^{a}$ contains the structure constants of a non-abelian algebra coupling the Yang-Mills fields, and it is antisymmetric on indices $b c$ :

$$
f_{b c}^{a}=f_{[b c]}^{a} \rightarrow f_{b c}^{a}=-f_{c b}^{a} .
$$

The expression $\delta a_{2}+\gamma a_{1}=\partial_{\mu} j^{\mu}$ is solved by taking the Koszul-Tate differential $\delta$ from (80):

$$
\begin{aligned}
\delta a_{2} & =\frac{1}{2} \delta\left(\eta_{a}^{*} f_{b c}^{a} \eta^{b} \eta^{c}\right) \\
& =-\frac{1}{2} \partial_{\mu}\left(A_{a}^{* \mu} f_{b c}^{a} \eta^{b} \eta^{c}\right)+\gamma\left(A_{a}^{* \mu} f_{b c}^{a} \eta^{b} A_{\mu}^{c}\right)
\end{aligned}
$$

We simply notice that

$$
\delta a_{2}-\gamma\left(A_{a}^{* \mu} f_{b c}^{a} \eta^{b} A_{\mu}^{c}\right)=-\frac{1}{2} \partial_{\mu}\left(A_{a}^{* \mu} f_{b c}^{a} \eta^{b} \eta^{c}\right) .
$$

This indicates

$$
a_{1}=-A_{a}^{* \mu} f_{b c}^{a} \eta^{b} A_{\mu}^{c}, \quad \stackrel{(1)}{j^{\mu}}=-\frac{1}{2} A_{a}^{* \mu} f_{b c}^{a} \eta^{b} \eta^{c} .
$$

To obtain $a_{0}$, we solve $\delta a_{1}+\gamma a_{0}=\partial_{\mu} j^{\mu}$ by taking the Koszul-Tate differential $\delta$ from $a_{1}$ :

$$
\begin{aligned}
\delta a_{1} & =\delta\left(-A_{a}^{* \mu} f_{b c}^{a} \eta^{b} A_{\mu}^{c}\right) \\
& =\partial_{\nu}\left(-F_{a}^{v \mu} f_{b c}^{a} \eta^{b} A_{\mu}^{c}\right)+\gamma\left(\frac{1}{2} F_{a}^{v \mu} f_{b c}^{a} A_{\nu}^{b} A_{\mu}^{c}\right)+\frac{1}{2} F_{a}^{v \mu} f_{b c}^{a} \eta^{b} F_{v \mu}^{c} .
\end{aligned}
$$

The last term in above relation vanishes, i.e. 


$$
F_{a}^{v \mu} f_{b c}^{a} \eta^{b} F_{v \mu}^{c}=0
$$

since

$$
\begin{aligned}
F_{a}^{v \mu} f_{b c}^{a} \eta^{b} F_{v \mu}^{c} & =\frac{1}{2} k_{a m} \sigma^{\nu \alpha} \sigma^{\mu \beta} F_{\alpha \beta}^{m} F_{v \mu}^{b} f_{b c}^{a} \eta^{c} \\
& =\frac{1}{2} \sigma^{\nu \alpha} \sigma^{\mu \beta} F_{\alpha \beta}^{m} F_{v \mu}^{b} f_{m b c} \eta^{c}=0,
\end{aligned}
$$

while

$$
f_{m b c}=k_{a m} f_{b c}^{a}, \quad f_{m b c}=-f_{b m c} .
$$

Therefore, we derive

$$
\delta a_{1}-\gamma\left(\frac{1}{2} F_{a}^{v \mu} f_{b c}^{a} A_{\nu}^{b} A_{\mu}^{c}\right)=\partial_{\nu}\left(-F_{a}^{v \mu} f_{b c}^{a} \eta^{b} A_{\mu}^{c}\right) .
$$

It shows

$$
a_{0}=-\frac{1}{2} F_{a}^{\nu \mu} f_{b c}^{a} A_{\nu}^{b} A_{\mu}^{c}, \quad \stackrel{(0)}{j^{\mu}}=-F_{a}^{\nu \mu} f_{b c}^{a} \eta^{b} A_{\mu}^{c} .
$$

The results for the first-order deformation are summarized as follows:

$$
a=-\frac{1}{2} F_{a}^{\nu \mu} f_{b c}^{a} A_{v}^{b} A_{\mu}^{c}-A_{a}^{* \mu} f_{b c}^{a} \eta^{b} A_{\mu}^{c}+\frac{1}{2} \eta_{a}^{*} f_{b c}^{a} \eta^{b} \eta^{c} .
$$

Finally, we derive

$$
S_{1}=\int \mathrm{d}^{D} x\left(-\frac{1}{2} F_{a}^{\nu \mu} f_{b c}^{a} A_{\nu}^{b} A_{\mu}^{c}-A_{a}^{* \mu} f_{b c}^{a} \eta^{b} A_{\mu}^{c}+\frac{1}{2} \eta_{a}^{*} f_{b c}^{a} \eta^{b} \eta^{c}\right) .
$$

The first-order deformations of the solution $\left(S_{1}\right)$ of the master equation were determined for the action (58). It is seen that gauge generators are field independent, and are reduced to a sum of terms with antighost numbers from zero to two.

\subsection{Higher-Order Deformations}

We now consider the higher-order deformations of the master equation for the action (50). The second-order deformation $\left(\lambda^{2}\right)$ of the master equation are determined from the solution $\left(S_{1}, S_{1}\right)+2 s S_{2}=0$. Let us assume that

$$
S_{2}=\int \mathrm{d}^{D} x b,
$$

that takes the local form

$$
\Delta+2 s b=\partial_{\mu} m^{\mu} .
$$

Using the Equation (88) from Section 5.1, we calculate $\left(S_{1}, S_{1}\right)$ :

$$
\begin{aligned}
\left(S_{1}, S_{1}\right) & \equiv \int \mathrm{d}^{D} x \Delta=\left(\int \mathrm{d}^{D} x a, \int \mathrm{d}^{D} y a\right) \\
& =\int \mathrm{d}^{D} x \mathrm{~d}^{D} y(a(x), a(x)),
\end{aligned}
$$

while employing the following relations

$$
\begin{gathered}
\left(\eta^{a}(x), \eta_{b}^{*}(y)\right)=\left(\eta_{b}^{*}(y), \eta^{a}(x)\right)=-\delta_{b}^{a} \delta^{D}(x-y), \\
\left(A_{\mu}^{a}(x), A_{b}^{* \nu}(y)\right)=\left(A_{b}^{* \nu}(y), A_{\mu}^{a}(x)\right)=-\delta_{b}^{a} \delta_{\mu}^{v} \delta^{D}(x-y),
\end{gathered}
$$

and the definitions 


$$
\begin{gathered}
\sigma^{\alpha \rho} \sigma^{\beta \lambda} k_{m g} f_{\rho \lambda}^{g} \equiv \sigma^{\alpha \rho} \sigma^{\beta \lambda} k_{m g}\left(\partial_{\rho} A_{\lambda}^{g}-\partial_{\lambda} A_{\rho}^{g}\right), \\
\int \mathrm{d}^{D} x \delta^{D}(x-y) f(x) \equiv f(y) .
\end{gathered}
$$

They lead to the following expression $\Delta$ :

$$
\begin{aligned}
\Delta= & -f_{e m}^{a} f_{n p}^{e} \eta_{a}^{*} \eta^{m} \eta^{n} \eta^{p}-\left(f_{e m}^{a} f_{n p}^{e}+f_{e n}^{a} f_{p m}^{e}+f_{e p}^{a} f_{m n}^{e}\right) A_{a}^{* \mu} \eta^{m} \eta^{n} A_{\mu}^{p} \\
& +\left(f_{e n}^{a} f_{p m}^{e}-f_{e m}^{a} f_{p n}^{e}\right) F_{a}^{\alpha \beta} A_{\alpha}^{m} A_{\beta}^{n} \eta^{p}+f_{b c}^{a} k_{m a} f_{n p}^{m} \sigma^{\alpha \rho} \sigma^{\beta \mu}\left(\partial_{\rho} \eta^{b}\right) A_{\mu}^{c} A_{\alpha}^{n} A_{\beta}^{p} \\
& +f_{b c}^{a} k_{m a} f_{n p}^{m} \sigma^{\alpha \rho} \sigma^{\beta \mu} \eta^{b}\left(\partial_{\rho} A_{\mu}^{c}\right) A_{\alpha}^{n} A_{\beta}^{p}-f_{b c}^{a} k_{m a} f_{n p}^{m} \sigma^{\alpha \mu} \sigma^{\beta \lambda}\left(\partial_{\lambda} \eta^{b}\right) A_{\mu}^{c} A_{\alpha}^{n} A_{\beta}^{p} \\
& -f_{b c}^{a} k_{m a} f_{n p}^{m} \sigma^{\alpha \mu} \sigma^{\beta \lambda} \eta^{b}\left(\partial_{\rho} A_{\mu}^{c}\right) A_{\alpha}^{n} A_{\beta}^{p},
\end{aligned}
$$

that is reduced to

$$
\begin{aligned}
\Delta= & -\frac{1}{3 !} f_{e[m}^{a} f_{n p]}^{e} \eta_{a}^{*} \eta^{m} \eta^{n} \eta^{p}-f_{e[m}^{a} f_{n p]}^{e} A_{a}^{* \mu} \eta^{m} \eta^{n} A_{\mu}^{p} \\
& -f_{e[m}^{a} f_{n p]}^{e} F_{a}^{\alpha \beta} A_{\alpha}^{m} A_{\beta}^{n} \eta^{p}+2 f_{b c}^{a} k_{m a} f_{n p}^{m} \sigma^{\alpha \rho} \sigma^{\beta \mu}\left(\partial_{\rho} \eta^{b}\right) A_{\mu}^{c} A_{\alpha}^{n} A_{\beta}^{p} .
\end{aligned}
$$

We then decompose $\Delta$ into the following terms,

$$
\Delta=\Delta_{0}+\Delta_{1}+\Delta_{2},
$$

namely,

$$
\begin{gathered}
\Delta_{0} \equiv-f_{e[m}^{a} f_{n p]}^{e} F_{a}^{\alpha \beta} A_{\alpha}^{m} A_{\beta}^{n} \eta^{p}+2 f_{b c}^{a} k_{m a} f_{n p}^{m} \sigma^{\alpha \rho} \sigma^{\beta \mu}\left(\partial_{\rho} \eta^{b}\right) A_{\mu}^{c} A_{\alpha}^{n} A_{\beta}^{p} . \\
\Delta_{1} \equiv-f_{e[m}^{a} f_{n p]}^{e} A_{a}^{* \mu} \eta^{m} \eta^{n} A_{\mu}^{p}, \\
\Delta_{2} \equiv-\frac{1}{3 !} f_{e[m}^{a} f_{n p]}^{e} \eta_{a}^{*} \eta^{m} \eta^{n} \eta^{p},
\end{gathered}
$$

We also define

$$
b \equiv b_{0}+b_{1}+b_{2} .
$$

From (91), it follows a set of equations

$$
\begin{gathered}
\Delta_{2}+2 \gamma b_{2}=\partial_{\mu} m^{\mu}, \\
\Delta_{1}+2 \delta b_{2}+2 \gamma b_{1}=\partial_{\mu} \stackrel{(1)}{m^{\mu},} \\
\Delta_{0}+2 \delta b_{1}+2 \gamma b_{0}=\partial_{\mu} \stackrel{(0)}{ }^{\mu} .
\end{gathered}
$$

Equations (99) and (101) imply

$$
\Delta_{2}=0, \quad b_{2}=0,
$$

and

$$
f_{e[m}^{a} f_{n p]}^{e}=0 .
$$

The later expression is called the Jacobi identity. Similarly, we obtain

$$
\Delta_{1}=0, \quad b_{1}=0 .
$$

So, the Equation (103) remains to be solved:

$$
2 f_{b c}^{a} k_{m a} f_{n p}^{m} \sigma^{\alpha \rho} \sigma^{\beta \mu}\left(\partial_{\rho} \eta^{b}\right) A_{\mu}^{c} A_{\alpha}^{n} A_{\beta}^{p}+2 \gamma b_{0}=\partial_{\mu}{ }^{(0)} m^{\mu} .
$$

We solve it by substituting the exterior longitudinal differential $\gamma$ of poten- 
tials $A_{\mu}^{a}\left(\gamma A_{\mu}^{a}=\partial_{\mu} \eta^{\alpha}\right)$ :

$$
2 f_{b c}^{a} k_{m a} f_{n p}^{m} \sigma^{\alpha \rho} \sigma^{\beta \mu}\left(\partial_{\rho} \eta^{b}\right) A_{\mu}^{c} A_{\alpha}^{n} A_{\beta}^{p}=\gamma\left(-\frac{1}{2} f_{b c}^{a} k_{a m} f_{n p}^{m} \sigma^{\alpha \rho} \sigma^{\beta \mu} A_{\rho}^{b} A_{\mu}^{c} A_{\alpha}^{n} A_{\beta}^{p}\right) .
$$

Accordingly, we derive

$$
b_{0}=-\frac{1}{4} f_{b c}^{a} k_{a m} f_{n p}^{m} \sigma^{\alpha \rho} \sigma^{\beta \mu} A_{\rho}^{b} A_{\mu}^{c} A_{\alpha}^{n} A_{\beta}^{p} .
$$

Hence, the second-order deformations becomes

$$
S_{2}=\int \mathrm{d}^{D} x\left(-\frac{1}{4} f_{b c}^{a} k_{a m} f_{n p}^{m} \sigma^{\alpha \rho} \sigma^{\beta \mu} A_{\rho}^{b} A_{\mu}^{c} A_{\alpha}^{n} A_{\beta}^{p}\right) .
$$

The Jacobi identity (105) obviously implies

$$
\left(S_{1}, S_{2}\right)=0 \rightarrow S_{3}=0 .
$$

Similarly, all deformations with orders higher than the second-order completely vanish:

$$
S_{k}=0, \forall k \geq 3 \text {. }
$$

As a result, the solution to the deformations becomes $S=S_{0}+\lambda S_{1}+\lambda^{2} S_{2}$, that corresponds to the following Yang-Mills theory:

$$
\begin{aligned}
S= & \int \mathrm{d}^{D} x\left(-\frac{1}{4} F_{\mu \nu}^{a} F_{a}^{\mu v}+A_{a}^{* \mu} \partial_{\mu} \eta^{a}\right) \\
& +\lambda \int \mathrm{d}^{D} x\left(-\frac{1}{2} F_{a}^{v \mu} f_{b c}^{a} A_{v}^{b} A_{\mu}^{c}-A_{a}^{* \mu} f_{b c}^{a} \eta^{b} A_{\mu}^{c}+\frac{1}{2} \eta_{a}^{*} f_{b c}^{a} \eta^{b} \eta^{c}\right) \\
& +\lambda^{2} \int \mathrm{d}^{D} x\left(-\frac{1}{4} f_{b c}^{a} k_{a m} f_{n p}^{m} \sigma^{\alpha \rho} \sigma^{\beta \mu} A_{\rho}^{b} A_{\mu}^{c} A_{\alpha}^{n} A_{\beta}^{p}\right) .
\end{aligned}
$$

We have determined the Yang-Mills theory from the first- and second-order deformations of the master equation. The solutions of the master equation, which entirely include the gauge structures, are decomposed into terms with the antighost numbers from zero to two. In other words, the part with the antighost number equal to zero represents the Lagrangian action, while the antighost number one is proportional to the gauge generators. The terms with higher antighost numbers provide the reducibility functions, where the on-shell relations become linear components in the ghosts for ghosts. It is shown that all functions with order higher than second vanish in this model.

\subsection{Interacting Theory}

Let us consider the Equation (109) and identify the entire gauge structure of the Lagrangian model that describes all consistent interactions in the $D$ -dimensional free Yang-Mills theory.

The antighost number zero of (109) shall provide the Lagrangian action of the interacting theory:

$$
\begin{aligned}
S_{0}^{L}\left[A_{\mu}^{a}\right]= & \int \mathrm{d}^{D} x\left(-\frac{1}{4} F_{\mu \nu}^{a} F_{a}^{\mu \nu}\right)+\lambda \int \mathrm{d}^{D} x\left(-\frac{1}{2} F_{a}^{\nu \mu} f_{b c}^{a} A_{v}^{b} A_{\mu}^{c}\right) \\
& +\lambda^{2} \int \mathrm{d}^{D} x\left(-\frac{1}{4} f_{b c}^{a} k_{a m} f_{n p}^{m} \sigma^{\alpha \rho} \sigma^{\beta \mu} A_{\rho}^{b} A_{\mu}^{c} A_{\alpha}^{n} A_{\beta}^{p}\right) .
\end{aligned}
$$


Accordingly, the Yang-Mills theory is characterized by the following non- abelian action:

$$
S_{0}^{L}\left[A_{\mu}^{a}\right]=\int \mathrm{d}^{D} x\left(-\frac{1}{4} \mathcal{F}_{\mu \nu}^{a} \mathcal{F}_{a}^{\mu v}\right),
$$

where the non-abelian field strengths $\mathcal{F}_{\mu v}^{a}$ is defined by

$$
\mathcal{F}_{\mu v}^{a}=F_{\mu v}^{a}+\lambda f_{b c}^{a} A_{\mu}^{b} A_{v}^{c}
$$

and $f_{b c}^{a}$ is the gauge-invariant that provides the gauge symmetry of the Yang-Mills theory as follows

$$
\bar{\delta}_{\varepsilon} A_{\mu}^{a}=\partial_{\mu} \varepsilon^{a}-\lambda f_{b c}^{a} \varepsilon^{b} A_{\mu}^{c} \equiv D_{\mu} \varepsilon^{a} .
$$

So, the commutator among the deformed gauge transformations becomes:

$$
\left[\bar{\delta}_{\varepsilon_{1}}, \bar{\delta}_{\varepsilon_{2}}\right] A_{\mu}^{a}=\bar{\delta}_{\varepsilon} A_{\mu}^{a}
$$

The gauge symmetry remains abelian to order $\lambda$, and satisfies the equation of motion

$$
D^{\mu} \mathcal{F}_{\mu v}^{a}=0
$$

The invariance of the action under the gauge transformations (113) is also obtained by the Noether identities

$$
D^{\mu}\left(\frac{\delta \mathcal{L}_{0}}{\delta A_{\mu}^{a}}\right) \equiv D^{\mu} D^{v} \mathcal{F}_{\mu \nu}^{a}=0 .
$$

The antighost number one of the deformation of the master equation allows to identify the gauge transformations (113) of the action (110) by substituting the ghost $\eta^{a}$ with gauge parameter $\varepsilon^{a}$. The antighost number two in (109) reads the complete gauge structure of the so-called interacting theory that determines the commutator (114) among the deformed gauge transformations.

\section{Conclusion}

In this paper, we reviewed deformed gauge transformations in the framework of the BRST-antifield formalism characterized by the antibracket that acts similar to the Poisson bracket in the Hamiltonian formalism. We provided the BRST cohomology of the consistent interactions through several order deformations of the master equation. The BRST-antifield formalism in the cohomological space provides the generalized framework of consistent interactions among fields with a gauge freedom by any types of invariant action. We see that higher order deformations could be neglected due to non local interactions and their obstruction of consistent local couplings, which are associated with the anomalous gauge quantization. We demonstrated its functions by applying the BRST-antifield formalism to the $D$-dimensional, free Yang-Mills theory. All deformations of the master equation for the massless Yang-Mills model were calculated by using the cohomological groups $H_{I}(s \mid d), I=0, \cdots, 2$, of the BRST differential. The first-order deformation is provided by the cohomological group $H_{1}(s \mid d)$, whereas the second-order deformation given by the cohomological group 
$\mathrm{H}_{2}(\mathrm{~s} \mid d)$ obstructs all higher-order deformations. The results show that the deformations can be synthesized by the conception that all orders higher than two are trivial, while gauge generators are imposed to be field independent, $H_{I}(s \mid d)=0, I>2$. The deformations stopped at the second-order of the coupling constants characterize the consistent interactions, which maintain the equation of motion, and provide the entire gauge structure of the interacting Yang-Mills theory.

\section{Acknowledgements}

The author thanks the editor and the referee for their comments. Research of A. Danehkar is funded by the EU contract MRTN-CT-2004-005104. This support is greatly appreciated.

\section{References}

[1] Dirac, P.A.M. (1950) Generalized Hamiltonian Dynamics. Canadian Journal of Mathematics, 2, 129-148. https://doi.org/10.4153/CJM-1950-012-1

[2] Dirac, P.A.M. (1958) The Theory of Gravitation in Hamiltonian Form. Proceedings of the Royal Society A Mathematical, Physical and Engineering Sciences, London, 246, 333-343. https://doi.org/10.1098/rspa.1958.0142

[3] Dirac, P. (1964) Lectures on Quantum Mechanics. Yeshiva University, New York.

[4] Anderson, J.L. and Bergmann, P.G. (1951) Constraints in Covariant Field Theories. Physical Review, 83, 1018-1025. https://doi.org/10.1103/PhysRev.83.1018

[5] Bergmann, P.G. and Goldberg, I. (1955) Dirac Bracket Transformations in Phase Space. Physical Review, 98, 531-538. https://doi.org/10.1103/PhysRev.98.531

[6] Weinberg, S. (1995) The Quantum Theory of Fields. Cambridge University Press, Cambridge. https://doi.org/10.1017/CBO9781139644167

[7] Gotay, M.J. and Nester, J.M. (1979) Presymplectic Lagrangian Systems. I: The Constraint Algorithm and the Equivalence Theorem. Annales de P Institut Henri Poincaré, 30, 129-142.

[8] Gotay, M.J. and Nester, J.M. (1980) Presymplectic Lagrangian Systems. II: The Second-Order Equation Problem. Annales de I Institut Henri Poincaré, 32, 1-13.

[9] Batlle, C., Gomis, J., Pons, J.M. and Roman-Roy, N. (1986) Equivalence between the Lagrangian and Hamiltonian Formalism for Constrained Systems. Journal of Mathematical Physics, 27, 2953-2962. https://doi.org/10.1063/1.527274

[10] Gitman, D.M. and Tyutin, I.V. (1990) Quantization of Fields with Constraints. Springer-Verlag, Berlin. https://doi.org/10.1007/978-3-642-83938-2

[11] Govaerts, J. (1991) Hamiltonian Quantisation and Constrained Dynamics. Leuven University Press, Leuven.

[12] Hanson, A., Regge, T. and Teitelboim, C. (1976) Constrained Hamiltonian Systems. Accademia Nazionale dei Lincei, Rome.

[13] Landau, L.D. and Lifshitz, E.M. (1976) Mechanics. Sykes, J.B. and Bell, J.S., Trans., Cambridge University Press, Cambridge.

[14] Sudarshan, E.C.G. and Mukunda, N. (1974) Classical Dynamics. Wiley, New York.

[15] Sundermeyer, K. (1982) Constrained Dynamics. Lecture Notes in Physics, Springer, Berlin.

[16] Becchi, C., Rouet, A. and Stora, R. (1974) The Abelian Higgs Kibble Model, Unitar- 
ity of the S-Operator. Physics Letters B, 52, 344-346. https://doi.org/10.1016/0370-2693(74)90058-6

[17] Becchi, C., Rouet, A. and Stora, R. (1975) Renormalization of the Abelian HiggsKibble Model. Communications in Mathematical Physics, 42, 127-162. https://doi.org/10.1007/BF01614158

[18] Becchi, C., Rouet, A. and Stora, R. (1976) Renormalization of Gauge Theories. Annals of Physics, 98, 287-321. https://doi.org/10.1016/0003-4916(76)90156-1

[19] Tyutin, I.V. (1975) Gauge Invariance in Field Theory and Statistical Physics in Operator Formalism. LEBEDEV-75-39. arXiv:0812.0580 [hep-th]

[20] Brandt, F., Dragon, N. and Kreuzer, M. (1989) All Consistent Yang-Mills Anomalies. Physics Letters B, 231, 263-270. https://doi.org/10.1016/0370-2693(89)90211-6

[21] Brandt, F., Dragon, N. and Kreuzer, M. (1989) All Solutions of the Consistency Equations. DESY 89-076, ITP-UH 2/89.

[22] Fisch, J., Henneaux, M., Stasheff, J. and Teitelboim, C. (1989) Existence, Uniqueness and Cohomology of the Classical BRST Charge with Ghosts of Ghosts. Communications in Mathematical Physics, 120, 379-407. https://doi.org/10.1007/BF01225504

[23] Henneaux, M. (1990) Lectures on the Antifield-BRST Formalism for Gauge Theories. Nuclear Physics B-Proceedings Supplements, 18, 47-105.

https://doi.org/10.1016/0920-5632(90)90647-D

[24] Batalin, I.A. and Vilkovisky, G.A. (1981) Gauge Algebra and Quantization. Physics Letters B, 102, 27-31. https://doi.org/10.1016/0370-2693(81)90205-7

[25] Batalin, I.A. and Vilkovisky, G.A. (1983) Quantization of Gauge Theories with Linearly Dependent Generators. Physical Review D, 28, 2567-2582.

https://doi.org/10.1103/PhysRevD.28.2567

[26] Batalin, I.A. and Vilkovisky, G.A. (1984) Erratum: Quantization of Gauge Theories with Linearly Dependent Generators. Physical Review D, 30, 508.

https://doi.org/10.1103/PhysRevD.30.508

[27] Fisch, J.M.L. and Henneaux, M. (1990) Homological Perturbation Theory and the Algebraic Structure of the Antifield-Antibracket Formalism for Gauge Theories. Communications in Mathematical Physics, 128, 627-640. https://doi.org/10.1007/BF02096877

[28] Henneaux, M. and Teitelboim, C. (1991) Quantization of Gauge Systems. Princeton University Press, Princeton.

[29] Gomis, J. and Paŕis, J. (1993) Field-Antifield Formalism for Anomalous Gauge Theories. Nuclear Physics B, 395, 288-324. hep-th/9204065 https://doi.org/10.1016/0550-3213(93)90218-E

[30] Gomis, J., Pars, J. and Samuel, S. (1995) Antibracket, Antifields and Gauge-Theory Quantization. Physics Reports, 259, 1-145. hep-th/9412228 https://doi.org/10.1016/0370-1573(94)00112-G

[31] Barnich, G. and Henneaux, M. (1993) Consistent Couplings between Fields with a Gauge Freedom and Deformations of the Master Equation. Physics Letters B, 311, 123-129. hep-th/9304057 https://doi.org/10.1016/0370-2693(93)90544-R

[32] Henneaux, M. (1998) Consistent Interactions between Gauge Fields: The Cohomological Approach. Contemporary Mathematics, 219, 93-109. hep-th/9712226 https://doi.org/10.1090/conm/219/03070

[33] Barnich, G., Brandt, F. and Henneaux, M. (1995) Local BRST Cohomology in the Antifield Formalism: II. Application to Yang-Mills theory. Communications in Mathematical Physics, 174, 93-116. hep-th/9405194

https://doi.org/10.1007/BF02099465 
[34] Bizdadea, C. (2000) On the Cohomological Derivation of Topological Yang-Mills Theory. Europhysics Letters, 52, 123-129. hep-th/0006218 https://doi.org/10.1209/epl/i2000-00413-7

[35] Cioroianu, E.M. and Sararu, S.C. (2005) Self-Interactions in a Topological BF-Type Model in D=5. Journal of High Energy Physics, 2005, JHEP07. hep-th/0508035 https://doi.org/10.1088/1126-6708/2005/07/056

[36] Bizdadea, C., Cioroianu, E.M., Danehkar, A., Iordache, M., Saliu, S.O. and Sararu, S.C. (2009) Consistent Interactions of Dual Linearized Gravity in $\mathrm{D}=5$ : Couplings with a Topological BF Model. European Physical Journal C, 63, 491-519. arXiv:hep-th/0908.2169 https://doi.org/10.1140/epjc/s10052-009-1105-0

[37] Barnich, G., Henneaux, M. and Tatar, R. (1994) Consistent Interactions between Gauge Fields and Local BRST Cohomology: The Example of Yang-Mills Models. International Journal of Modern Physics D, 3, 139-144. hep-th/9307155 https://doi.org/10.1142/S0218271894000149

[38] Bizdadea, C., Ciobirca, C.C., Cioroianu, E.M., Saliu, S.O. and Sararu, S.C. (2003) Hamiltonian BRST Deformation of a Class of N-Dimensional BF-Type Theories. Journal of High Energy Physics, 2003, JHEP01. hep-th/0302037 https://doi.org/10.1088/1126-6708/2003/01/049

[39] Barnich, G., Brandt, F. and Henneaux, M. (2000) Local BRST Cohomology in Gauge Theories. Physics Reports, 338, 439-569. hep-th/0002245 https://doi.org/10.1016/S0370-1573(00)00049-1 


\section{Appendix}

\section{Antibracket Structure}

For a function $X(\psi)$ in a generic space, commutative or anticommutative, we state:

$$
\frac{\partial_{l} X}{\partial \psi}=\frac{\vec{\partial}}{\partial \psi} X, \frac{\partial_{r} X}{\partial \psi}=X \frac{\grave{\partial}}{\partial \psi} .
$$

The left derivative $\partial_{l}$ is an ordinary derivative (left to right). The right derivative $\partial_{r}$ is the derivative action from right to left.

For any $X(\psi)$ in a generic space, we get

$$
\frac{\partial_{l} X}{\partial \psi}=(-1)^{\varepsilon_{\psi}\left(\varepsilon_{X}+1\right)} \frac{\partial_{r} X}{\partial \psi} .
$$

Considering Equation (32) and Equation (118), it follows that

$$
(X, Y)=-(-1)^{\left(\varepsilon_{X}+1\right)\left(\varepsilon_{Y}+1\right)}(Y, X) \text {. }
$$

Assuming $X=Y$, one can find

$$
\frac{\partial_{r} X}{\partial \Phi^{A}} \frac{\partial_{l} X}{\partial \Phi_{A}^{*}}=(-1)^{\left(\varepsilon_{X}+1\right)\left(\varepsilon_{X}+1\right)} \frac{\partial_{r} X}{\partial \Phi_{A}^{*}} \frac{\partial_{l} X}{\partial \Phi^{A}} .
$$

For bosonic (commutative) and fermionic (anticommutative) variables, we have

$$
(X, X)=\left\{\begin{array}{cc}
2 \frac{\partial_{r} X}{\partial \Phi^{A}} \frac{\partial_{l} X}{\partial \Phi_{A}^{*}} & X \text { is commutative } \\
0 & X \text { is anticommutative. }
\end{array}\right.
$$

For any $X$, we have

$$
((X, X), X)=0, \quad \forall X
$$

Furthermore, the antibracket has the following properties:

$$
\begin{gathered}
(X, Y Z)=(X, Y) Z+(-1)^{\varepsilon_{Y} \varepsilon_{Z}}(X, Z) Y, \\
(X Y, Z)=X(Y, Z)+(-1)^{\varepsilon_{X} \varepsilon_{Y}} Y(X, Z), \\
((X, Y), Z)+(-1)^{\left(\varepsilon_{X}+1\right)\left(\varepsilon_{Y}+\varepsilon_{Z}\right)}((Y, Z), X)+(-1)^{\left(\varepsilon_{Z}+1\right)\left(\varepsilon_{X}+\varepsilon_{Y}\right)}((Z, X), Y)=0 .
\end{gathered}
$$


Submit or recommend next manuscript to SCIRP and we will provide best service for you:

Accepting pre-submission inquiries through Email, Facebook, LinkedIn, Twitter, etc. A wide selection of journals (inclusive of 9 subjects, more than 200 journals)

Providing 24-hour high-quality service

User-friendly online submission system

Fair and swift peer-review system

Efficient typesetting and proofreading procedure

Display of the result of downloads and visits, as well as the number of cited articles Maximum dissemination of your research work

Submit your manuscript at: http://papersubmission.scirp.org/

Or contact jhepgc@scirp.org 